Pacific Journal of Mathematics

ON REPRESENTATIONS OF CERTAIN SEMIGROUPS 


\title{
ON REPRESENTATIONS OF CERTAIN SEMIGROUPS
}

\author{
Michael FRIEDBERG
}

\begin{abstract}
A theory of representations for compact semigroups has been lacking due in large part to the absence of a translationinvariant carrying measure that exists for compact groups. The object in this paper is to show that for a compact, groupextremal affine semigroup there is a sufficient system of representations by linear operators on finite-dimensional complex linear spaces; in the abelian case, a sufficient system of affine semicharacters is obtained. As a result, a compact groupextremal affine semigroup is the inverse limit of compact, finite-dimensional, group-extremal affine semigroups.
\end{abstract}

A subset $S$ of a locally convex topological linear space $X$ (over the reals or complexes) will be called an affine semigroup if:

(1) $S$ is convex.

(2) There is an associative multiplication defined in $S$ which is jointly continuous in the topology on $S$ inherited from $X$.

(3) For fixed $x \in S$ the functions $y \rightarrow y x$ and $y \rightarrow x y$ are affine functions of $S$ into $S$.

In this paper, $S$ will always be compact. By a theorem due to Wendel [2], if $S$ is a compact affine semigroup with identity $u$, then each point of $S$ with inverse is an extreme point of $S$. If, conversely, each extreme point has an inverse then the set of extreme points of $S$ is the maximal group of the idempotent $u$ and is, therefore, compact [9]. In this case, we shall say $S$ is group-extremal.

Following [2], we will say two affine semigroups $S$ and $T$ are equivalent if there exists a bicontinuous isomorphism of $S$ onto $T$ which is also an affine function.

DeFinition 1. A representation of an affine semigroup $S$ is a function $P$ from $S$ to $B(M)$ the set of bounded linear operator on some finite-dimensional complex linear space $M$ satisfying:

(a) $P$ is continuous (with any locally convex topology on $B(M)$, all of which are equivalent).

(b) $P$ is a homomorphism.

(c) $P$ is affine.

DEFINITION 2. An affine semicharacter on $S$ is any complex-valued continuous affine homomorphism defined on $S$. We point out that if $S$ is compact and $f$ is any affine semicharacter on $S$ then $|f(x)| \leqq 1$ for each $x \in S$. 
In the remainder of this paper, $S$ will be a compact, group-extremal affine semigroup with identity $u$, and whose extreme points form the compact topological group $G$.

1. Representations of $S$. In this section, we shall prove the following:

THEOREM 1. For $x_{0}, y_{0} \in S, x_{0} \neq y_{0}$ there exists a representation $P$ of $S$ in $B(M), M$ a finite-dimensional complex linear space, satisfying

(1) $P\left(x_{0}\right) \neq P\left(y_{0}\right)$.

(2) $P^{*}(\sigma) \in P(S)$ for all $\sigma \in S$ (where $P^{*}(\sigma)$ is the adjoint of the operator $P(\sigma))$.

Many of the details of the proof are quite similar to those in group representations (cf. [1], [6], [7]) but we shall include them for the sake of completeness. By $C(S)(C(G))$ we mean the collection of all complex-valued continuous functions on $S(G)$. The supremum norm in $C(S)$ is denoted by $\|\cdot\|$ and in $C(G)$ by $\|\cdot\|_{*} \cdot A(S)$ will denote the norm closed subspace of $C(S)$ consisting of all affine continuous complex-valued functions. $A(G)$ denotes the set of restrictions to $G$ of elements of $A(S)$.

Lemma 1.1. (a) $A(G)$ is a closed subspace of $C(G)$.

(b) If $f, g \in A(S)$ and $f(x)=g(x)$ for $x \in G$ then $f(x)=g(x)$ for all $x \in S$.

(c) If $f_{n} \in A(G), g_{n} \in A(S)$ for $n=0,1,2, \cdots$ if $f_{n}(x)=g_{n}(x)$ for $x \in G, n=0,1,2,3, \cdots$ and if $\left\|f_{n}-f_{0}\right\|_{*} \rightarrow 0$ then $\left\|g_{n}-g_{0}\right\| \rightarrow 0$.

Proof of (a). Let $f_{n} \rightarrow f$ where $f_{n} \in A(G), n=1,2,3, \cdots$ and $f \in C(G)$. There exist $g_{n} \in A(S)$ such that $g_{n}(x)=f_{n}(x)$ for $x \in G$. For $\varepsilon>0$ there exists an $N$ such that if $m, n \geqq N$ and $x \in G$ then $\left|f_{n}(x)-f_{m}(x)\right|<\varepsilon / 2$. If $x_{1}, \cdots, x_{r} \in G, \lambda_{i} \geqq 0, \sum_{i=1}^{r} \lambda_{i}=1$ and $x=\sum_{i=1}^{r} \lambda_{i} x_{i}$ then

$$
\begin{aligned}
\left|g_{n}(x)-g_{m}(x)\right| & =\left|\sum_{i=1}^{r} \lambda_{i}\left[g_{n}\left(x_{i}\right)-g_{m}\left(x_{i}\right)\right]\right| \\
& =\left|\sum_{i=1}^{r} \lambda_{i}\left[f_{n}\left(x_{i}\right)-f_{m}\left(x_{i}\right)\right]\right|<\frac{\varepsilon}{2} .
\end{aligned}
$$

Since $g_{n}-g_{m}$ is continuous on $S$, and the elements $x$ of the above form are dense in $S$ [4], we have $\left|g_{n}(x)-g_{m}(x)\right|<\varepsilon$ for $x \in S$. Thus, $\left\{g_{n}\right\}_{n=1}^{\infty}$ is a Cauchy sequence in $C(S)$ and, hence, converges to $g \in C(S)$. Since $A(S)$ is clearly closed, $g \in A(S)$. Now for $x \in G, f_{n}(x) \rightarrow f(x)$ but $f_{n}(x)=g_{n}(x) \rightarrow g(x)$ so that $f(x)=g(x)$ and $f \in A(G)$.

Proof of (b). An application of the Krein-Milman Theorem. 
Proof of (c). By an argument similar to the proof of (a) $\left\|g_{n}-h\right\| \rightarrow 0$ for some $h \in A(S)$. But $f_{n}(x)=g_{n}(x)$ for all $x \in G$ so that $h(x)=f_{0}(x)=g_{0}(x)$ for $x \in G$. By $(b), h(x)=g_{0}(x)$ for all $x \in S$.

Proof of theorem. By $L^{2}(G)$, we mean the Hilbert space of all functions on $G$ which are square-integrable with respect to Haar measure on $G$, where the inner product is defined as usual. (i.e. $(f, g)=\int_{1 / 2} f \bar{g} d x$ ). We denote the norm of an element $f \in L^{2}(G)$ by $\|f\|_{2}=\left(\int|f|^{2} d x\right)^{1 / 2}$.

We now fix $x_{0}, y_{0} \in S$ where $x_{0} \neq y_{0}$. There exists a set $U$ which is open in $G, u \in U$, and $\langle U\rangle x_{0} \cap\langle U\rangle y_{0}=\varnothing . \quad(\langle U\rangle$ denotes the closed convex hull of $U)$. This follows from $u x_{0} \neq u y_{0}$, the continuity of multiplication in $S$, and the local convexity of the containing space $X$.

There exists a real-valued function $f_{0} \in A(S)$ satisfying:

$$
\min _{z \in\langle U\rangle x_{0}}\left\{f_{0}(z)\right\}>\max _{z \in\left\langle U>y_{0}\right.}\left\{f_{0}(z)\right\}
$$

[3]. Choose $h \in C(G), h(u)=1, h=0$ in $G \backslash U$, and $0 \leqq h \leqq 1$. For $z \in G$, let

$$
k(z)=\frac{h(z)+h\left(z^{-1}\right)}{2}
$$

then $k \in C(G), 0 \leqq k \leqq 1, k(u)=1, k=0$ in $G \backslash U$ and $k(z)=k\left(z^{-1}\right)$. We then have

$$
\begin{aligned}
\int k\left(z^{-1}\right) f_{0}\left(z x_{0}\right) d z & =\int_{U} k\left(z^{-1}\right) f_{0}\left(z x_{0}\right) d z>\int_{\sigma} k\left(z^{-1}\right) f_{0}\left(z y_{0}\right) d z \\
& =\int k\left(z^{-1}\right) f_{0}\left(z y_{0}\right) d z
\end{aligned}
$$

Hence,

(1) $\int k\left(z^{-1}\right) f_{0}\left(z x_{0}\right) d z \neq \int k\left(z^{-1}\right) f_{0}\left(z y_{0}\right) d z$

The operator in $L^{2}(G)$ defined by

(2) $T f(x)=\int k\left(z^{-1}\right) f(z x) d z$ for $f \in L^{2}(G), x \in G$ takes $L^{2}(G)$ into $C(G)$ and is a completely continuous, symmetric bounded linear operator in $L^{2}(G)$ [8; p. 242]. Further, $\|T f\|_{*} \leqq\|k\|_{2} \cdot\|f\|_{2}$ so that $f \rightarrow T f$ is continuous in the norm topology on $C(G)$. If $f \in A(G)$ then there is a $g \in A(S)$ such that $g(x)=f(x)$ for $x \in G$. If we define:

(3) $g^{\prime}(x)=\int k\left(z^{-1}\right) g(z x) d z$ then $g^{\prime} \in A(S)$ and for

$$
x \in G, g^{\prime}(x)=\int k\left(z^{-1}\right) g(z x) d z=\int k\left(z^{-1}\right) f(z x) d z=T f(x) .
$$

Thus, if $f \in A(G)$, then $T f \in A(G)$. If we let $H$ denote the closure of 
$A(G)$ in $L^{2}(G)$, then $H$ is a closed invariant subspace of $T$. In fact, if $f \in H$, there exists a sequence $f_{n} \in A(G)$ such that $\left\|f_{n}-f\right\|_{2} \rightarrow 0$. But then $\left\|T f_{n}-T f\right\|_{*} \rightarrow 0$ and since $T f_{n} \in A(G)$, which is norm closed in $C(G)$, we have $T f \in A(G)$. Hence, $T$ takes $H$ into $A(G)$. Using $T$ again to denote the restriction of $T$ to $H$, we have again that $T$ is a completely-continuous, symmetric bounded linear operator in $H$. By a well-known theorem (cf. [8; p. 233]) there exists a sequence $\left\{\psi_{i}\right\}_{i=1}^{\infty}$ where

(4) $\psi_{i} \in H$ for $i=1,2, \cdots$

(5) $T \psi_{i}=\lambda_{i} \psi_{i}$ for some real number $\lambda_{i} \neq 0$

(6) $\left(\psi_{i}, \psi_{j}\right)=\delta_{i j}\left(\delta_{i j}\right.$ is the Kronecker delta function)

(7) $T f=\sum_{i=1}^{\infty}\left(T f, \psi_{i}\right) \psi_{i}$ for each $f \in H$ and where the series converges in $L^{2}(G)$ norm.

(8) For each $\lambda \neq 0, M_{\lambda}=\{f \in H: T f=\lambda \cdot f\}$ is finite-dimensional. Note that $\psi_{i}=T\left(\left(1 / \lambda_{i}\right) \psi_{i}\right)$ and since $\left(1 / \lambda_{i}\right) \psi_{i} \in H$, it follows that $\psi_{i} \in A(G)$. Also, using a computation that can be found in $[1 ; \mathrm{p} .209]$ the series in (7) converges to $T f$ in the supremum norm on $C(G)$.

Now since $\psi_{i} \in A(G)$ for each $i$, there exists $\hat{\psi}_{i} \in A(S)$ such that $\hat{\psi}_{i}(x)=\psi_{i}(x)$ for $x \in G$. Further, if $g \in A(S)$ and $f$ denotes the restriction of $g$ to $G$ then $f \in A(G)$ so that $T f=\sum_{i=1}^{\infty}\left(T f, \psi_{i}\right) \psi_{i}$ where the series converges in supremum norm on $C(G)$. As in (3), if $g^{\prime}(x)=$ $\int k\left(z^{-1}\right) g(z x) d z$ for $x \in S$ then $g^{\prime} \in A(S)$ and for $x \in G, g^{\prime}(x)=T f(x)$. Also for $x \in G, n \geqq 1, \sum_{i=1}^{n}\left(T f, \psi_{i}\right) \hat{\psi}_{i}(x)=\sum_{i=1}^{n}\left(T f, \psi_{i}\right) \psi_{i}(x)$ and, hence, Lemma 1.1(c) implies that $g^{\prime}=\sum_{i=1}^{\infty}\left(T f, \psi_{i}\right) \hat{\psi}_{i}$ where the series converges in $A(S)$. In particular, if $f_{0}$ is our original function (1) and $g_{0}$ is the restriction to $G$ of $f_{0}$ then $f_{0}^{\prime}=\sum_{i=1}^{\infty}\left(T g_{0}, \psi_{i}\right) \hat{\psi}_{i}$. But by (1), $f_{0}^{\prime}\left(x_{0}\right) \neq f_{0}^{\prime}\left(y_{0}\right)$ so that for some $i, \hat{\psi}_{i}\left(x_{0}\right) \neq \hat{\psi}_{i}\left(y_{0}\right)$.

For $\lambda=\lambda_{i}, M_{\lambda}=\{f \in H, T f=\lambda \cdot f\}$ is a finite-dimensional subspace of $H$; hence, by Lemma $1.1(b) N_{\lambda}=\left\{f \in A(S): f^{\prime}=\lambda f\right\}$ is a finite-dimensional subspace of $A(S)$, and there exists $\hat{\psi}_{i} \in N_{\lambda}$ for which $\hat{\psi}_{i}\left(x_{0}\right) \neq \hat{\psi}_{i}\left(y_{0}\right) . \quad N_{\lambda}$ is easily seen to be a finite-dimensional Hilbert space with inner product again $(f, g)=\int f \bar{g} d x$. In fact, if $f \in A(S)$ and $(f, f)=0$ then $\int|f|^{2} d x=0$ so that $f(x)=0$ for $x \in G$. By Lemma 1.1(b), $f(x)=0$ for all $x \in S$. For $f \in N_{\lambda}$, it is easily seen $\left(|\lambda| /\|k\|_{2}\right)\|f\| \leqq(f, f)^{1 / 2} \leqq\|f\|$ so that $N_{\lambda}$ is complete with respect to this inner product. For $\sigma \in S$, we define the linear operator $P(\sigma)$ in $N_{\lambda}$ by:

(9) $[P(o) f](x)=f(x \sigma)$ where $f \in N_{\lambda}, x \in S$. We have

$$
\begin{aligned}
{[P(\sigma) f]^{\prime}(x) } & =\int k\left(z^{-1}\right) P(\sigma) f(z x) d z=\int k\left(z^{-1}\right) f(z x \sigma) d z \\
& =f^{\prime}(x \sigma)=\lambda f(x \sigma)=\lambda[P(\sigma) f](x) .
\end{aligned}
$$


Hence, $P(\sigma)$ clearly takes $N_{\lambda}$ to $N_{\lambda}$. It is clear that the map $\sigma \rightarrow P(\sigma)$ is continuous in the strong operator topology. Further, $[P(\sigma \tau) f](x)=$ $f(x \sigma \tau)=P(\sigma)[P(\tau) f](x)$ so that $P(\sigma \tau)=P(\sigma) P(\tau)$ and $\sigma \rightarrow P(\delta)$ is a homomorphism. For $\sigma, \tau \in S 0 \leqq \lambda \leqq 1$ and $x \in S$ we have

$$
\begin{aligned}
{[P(\lambda \sigma+(1-\lambda) \tau) f](x) } & =f(x[\lambda \sigma+(1-\lambda) \tau]) \\
& =\lambda f(x \sigma)+(1-\lambda) f(x \tau) \\
& =[\lambda P(\sigma)+(1-\lambda) P(\tau) f](x)
\end{aligned}
$$

and $\sigma \rightarrow P(\sigma)$ is now an affine continuous homomorphism of $S$ into the bounded linear operators on the finite-dimensional space $N_{\lambda}$.

Note further that there exists $\hat{\psi}_{i} \in N_{\lambda}$ where $\hat{\psi}_{i}\left(x_{0}\right) \neq \hat{\psi}_{i}\left(y_{0}\right)$. Then $\left[P\left(x_{0}\right) \hat{\psi}_{i}\right](u)=\hat{\psi}_{i}\left(x_{0}\right) \neq \hat{\psi}_{i}\left(y_{0}\right)=\left[P\left(y_{0}\right) \hat{\psi}_{i}\right](u)$ and $P\left(x_{0}\right) \neq P\left(y_{0}\right)$. Finally, for $x \in G, f, g \in N_{\lambda}$

$$
\begin{aligned}
(P(x) f, g) & =\int[P(x) f](y) \overline{g(y)} d y=\int f(y x) \overline{g(y)} d y \\
& =\int f(y) \overline{g\left(y x^{-1}\right)} d y=\int f(y) \overline{\left[P\left(x^{-1}\right) g\right]}(y) d y=\left(f, P\left(x^{-1}\right) g\right) .
\end{aligned}
$$

Hence, we have for $x \in G, P^{*}(x)=P\left(x^{-1}\right)$. If $x_{1}, x_{2}, \cdots x_{n} \in G, \lambda_{i} \geqq 0$, $\sum_{i=1}^{n} \lambda_{i}=1$ and $x=\sum_{i=1}^{n} \lambda_{i} x_{i}$ then

$$
P^{*}(x)=\sum_{i=1}^{n} \lambda_{i} P^{*}\left(x_{i}\right)=\sum_{i=1}^{n} \lambda_{i} P\left(x_{i}^{-1}\right)=P\left(\sum_{i=1}^{n} \lambda_{i} x_{i}^{-1}\right) \in P(S) .
$$

Since $P(S)$ is compact and convex, it follows by continuity of $P$ and the Krein-Milman Theorem that $P^{*}(\sigma) \in P(S)$ for each $\sigma \in S$ and the proof is complete.

CoRollary 1.1. If $G$ is metrizable, there is a countable number of representations which separate points.

Proof. In Theorem 1, to separate two points we obtained a neighborhood of the identity, and then constructed a countable number of representations using this neighborhood. It is clear this neighborhood may be taken from a countable basis at the identity, giving rise to a countable number of representations which separate the points of $S$.

2. Affine semicharacters. In this section, we assume the additional condition that $S$ is abelian; then we have:

THEOREM 2. If $x_{0}, y_{0} \in S, x_{0} \neq y_{0}$ there exists an affine semicharacter $p$ such that $p\left(x_{0}\right) \neq p\left(y_{0}\right)$. 
Proof. By Theorem 1, there exists a representation $P$ of $S$ in the bounded linear operators $B(M)$ on the $n$-dimensional complex vector space $M$ for which $P\left(x_{0}\right) \neq P\left(y_{0}\right)$ and $P^{*}(\sigma) \in P(S)$ for each $\sigma \in S$. The space $M$ is then a finite-dimensional space invariant under the abelian family of operators $\{P(\sigma): \sigma \in S\}$ satisfying $P^{*}(\sigma) \in P(S)$ for $\sigma \in S$ and, hence, is spanned by one dimensional invariant subspaces. We thus obtain a basis $e_{1}, \cdots, e_{n}$ for $M$ where $P(\sigma) e_{i}=P_{i}(\sigma) e_{i}$ for each $i=$ $1,2, \cdots, n$ and $p_{i}(\sigma)$ is a complex number. The functions $p_{i}, \cdots, p_{n}$ are easily seen to be affine semicharacters of $S$. Since $P(x) \neq P(y)$, $p_{i}(x) \neq p_{i}(y)$ for some integer $i$ and we are finished. Using the representations of $S$ and the fact that they are affine maps we have:

THEOREM 3. A group-extremal affine semigroup is equivalent to the inverse limit of finite-dimensional group-extremal affine semigroups.

The proof of this theorem is completely analogous to the proof of the well-known theorem that a compact group is the inverse limit of compact Lie groups, so we shall omit it.

\section{BIBLIOGRAPHY}

1. C. Chevalley, Theory of Lie Groups, Princeton University Press, Princeton, 1946. 2. H. Cohen and H. S. Collins, Affine semigroups, Trans. Amer. Math. Soc. 93 (1959), 97-113.

3. J. L. Kelly and I. Namicka, Linear Topological Spaces, D. Van Nostrant, Princeton, 1963.

4. M. Krein and D. Milman, On extreme points of regular convex sets, Studia Math. 9 (1940).

5. K. de Leeuw and I. Glicksberg, Applications of Almost Period Conpactifications, Stanford University Press, Stanford, 1959.

6. D. Montgomery and L. Zippin, Topological Transformation Groups, Interscience Publishers, Inc., New York, 1955.

7. L. Pontrjagin, Topologische Gruppen, B. G. Teubner Verlagsgesellschaft, Leipzig, 1957.

8. F. Riesz and B. Sz.-Nagy, Functional Analysis, F. Ungar Publishing Co., New York, 1955.

9. A. D. Wallace, The structure of topological semigroups, Bull. Amer. Math. Soc. 61 (1955), 95-112.

Received August 2, 1965. The author would like to express his appreciation to Professor R.J. Koch for his advice in the preparation of this paper. This research was supported in part by National Science Foundation Grant No. GP-1637.

LOUISIANA STATE University

BATON ROUGE, LOUISIANA 


\title{
PACIFIC JOURNAL OF MATHEMATICS
}

\author{
EDITORS
}

\section{H. SAMELSON}

Stanford University

Stanford, California

J. P. JANS

University of Washington

Seattle, Washington 98105
J. DugundJI

University of Southern California

Los Angeles, California 90007

RICHARD ARENS

University of California

Los Angeles, California 90024

\section{ASSOCIATE EDITORS}

\section{E. F. BECKENBACH}

B. H. NEUMANN

F. WOLF

K. YosidA

\section{SUPPORTING INSTITUTIONS}

\author{
UNIVERSITY OF BRITISH COLUMBIA \\ CALIFORNIA INSTITUTE OF TECHNOLOGY \\ UNIVERSITY OF CALIFORNIA \\ MONTANA STATE UNIVERSITY \\ UNIVERSITY OF NEVADA \\ NEW MEXICO STATE UNIVERSITY \\ OREGON STATE UNIVERSITY \\ UNIVERSITY OF OREGON \\ OSAKA UNIVERSITY \\ UNIVERSITY OF SOUTHERN CALIFORNIA
}

\author{
STANFORD UNIVERSITY \\ UNIVERSITY OF TOKYO \\ UNIVERSITY OF UTAH \\ WASHINGTON STATE UNIVERSITY \\ UNIVERSITY OF WASHINGTON \\ AMERICAN MATHEMATICAL SOCIETY \\ CHEVRON RESEARCH CORPORATION \\ TRW SYSTEMS \\ NAVAL ORDNANCE TEST STATION
}

Mathematical papers intended for publication in the Pacific Journal of Mathematics should be typewritten (double spaced). The first paragraph or two must be capable of being used separately as a synopsis of the entire paper. It should not contain references to the bibliography. Manuscripts may be sent to any one of the four editors. All other communications to the editors should be addressed to the managing editor, Richard Arens at the University of California, Los Angeles, California 90024.

50 reprints per author of each article are furnished free of charge; additional copies may be obtained at cost in multiples of 50 .

The Pacific Journal of Mathematics is published monthly. Effective with Volume 16 the price per volume (3 numbers) is $\$ 8.00$; single issues, $\$ 3.00$. Special price for current issues to individual faculty members of supporting institutions and to individual members of the American Mathematical Society: $\$ 4.00$ per volume; single issues $\$ 1.50$. Back numbers are available.

Subscriptions, orders for back numbers, and changes of address should be sent to Pacific Journal of Mathematics, 103 Highland Boulevard, Berkeley 8, California.

Printed at Kokusai Bunken Insatsusha (International Academic Printing Co., Ltd.), No. 6, 2-chome, Fujimi-cho, Chiyoda-ku, Tokyo, Japan.

PUBLISHED BY PACIFIC JOURNAL OF MATHEMATICS, A NON-PROFIT CORPORATION

The Supporting Institutions listed above contribute to the cost of publication of this Journal, but they are not owners or publishers and have no responsibility for its content or policies. 


\section{Pacific Journal of Mathematics \\ Vol. 19, No. $2 \quad$ June, 1966}

Leonard Daniel Baumert, Extreme copositive quadratic forms . ......... 197

Fred James Bellar, Jr., Pointwise bounds for the second initial-boundary value problem of parabolic type ........................ 205

L. Carlitz and David Paul Roselle, Restricted bipartite partitions ........ 221

Robin Ward Chaney, On the transformation of integrals in measure space ........................................... 229

Colin W. Clark, An embedding theorem for function spaces ........... 243

Edwin Duda, A theorem on one-to-one mappings................ 253

Ben Fitzpatrick, Jr. and Donald Reginald Traylor, Two theorems on metrizability of Moore spaces.......................... 259

Allen Roy Freedman, An inequality for the density of the sum of sets of vectors in $n$-dimensional space ....................... 265

Michael Friedberg, On representations of certain semigroups .......... 269

Robert William Gilmer, Jr., The pseudo-radical of a commutative ring . . . . 275

Hikosaburo Komatsu, Fractional powers of operators ............... 285

Daniel Rider, Transformations of Fourier coefficients ................. 347

David Alan Sánchez, Some existence theorems in the calculus of variations ...................................... 357

Howard Joseph Wilcox, Pseudocompact groups............... 365

William P. Ziemer, Some lower bounds for Lebesgue area ............. 381 